\title{
Triplet and Singlet Excitations in the Valence Bond Crystal Phase of Kagome Lattice Heisenberg Model
}

\author{
Rajiv R. P. Singh \\ Department of Physics, University of California, Davis, CA 95616, USA \\ David A. Huse \\ Department of Physics, Princeton University, Princeton, NJ 08544, USA
}

(Dated: November 3, 2018)

\begin{abstract}
A proposed ground state for the Kagome Lattice Heisenberg Model (KLHM) consists of a Valence Bond Crystal (VBC) with a 36-site unit cell. We calculate the low-lying triplet and singlet excitations in the VBC phase for the infinite-lattice model and for the 36-site cluster. For the infinite lattice, the lowest triplet excitation is found to have a spin gap of approximately $0.08 \pm 0.02 J$ and a bandwidth of only about $0.01 \mathrm{~J}$. For the 36 -site cluster, consisting of a single unit cell with periodic boundary conditions, there are substantial finite-size effects: the spin gap there is estimated to be approximately $0.2 \mathrm{~J}$, close to the exact diagonalization result of $0.164 \mathrm{~J}$. The triplet excitations attract one another and form many bound states in the spin-singlet channel. We find a large number of such bound states for the 36-site cluster, many of which appear to lie below the spin gap, again in agreement with the results from exact diagonalization.
\end{abstract}

PACS numbers: $75.10 . \mathrm{Jm}$

\section{INTRODUCTION}

Ground state properties of the Kagome-Lattice Heisenberg Model have been studied by a wide variety of numerical and analytical techniques $1,2,2,3,4,5,6,7,8$, 9, 10, 11, 12, 13, 14, 15, 16, 17]. Very recently, we used [18] series expansion methods to make the case that a Valence Bond Crystal (VBC) phase consisting of a honeycomb lattice of 'perfect hexagons' with a 36-site unit cell (shown in Fig. 1) is the correct ground state phase of this model. We found the ground state energy of this state to be $-0.433 \pm 0.001 \mathrm{~J}$, which is lower than other existing variational estimates. We also showed that with periodic boundary conditions on the 36 -site cluster the energy of the state is further lowered by an amount that makes it compatible with the result from exact diagonalization.

One of the most intriguing features of the model is the finding of a large number of singlet states below the lowest triplet in the exact diagonalization studies of finite clusters [7, 8, $9,10,11]$. The VBC phase, with a large unit cell, provides a natural starting point for explaining these states. In the 36 -site cluster, the broken symmetries of the VBC phase would give rise to 48 lowlying singlet states for every singlet state in the brokensymmetry phase. However, there are several respects in which such an explanation has so far been unsatisfactory. First, the exact diagonalization studies do not see a clear separation between a 'tower' of ground states, and the other singlet states; the spectrum appears to be a continuum. Second, if a triplet is the lowest excitation in the VBC phase, one would expect only 48 singlets below the lowest triplet, whereas the number of such states is found to be about 200. Third, the quantum numbers of the lowest-lying states of the 36-site cluster do not match with the quantum numbers of the states that arise from

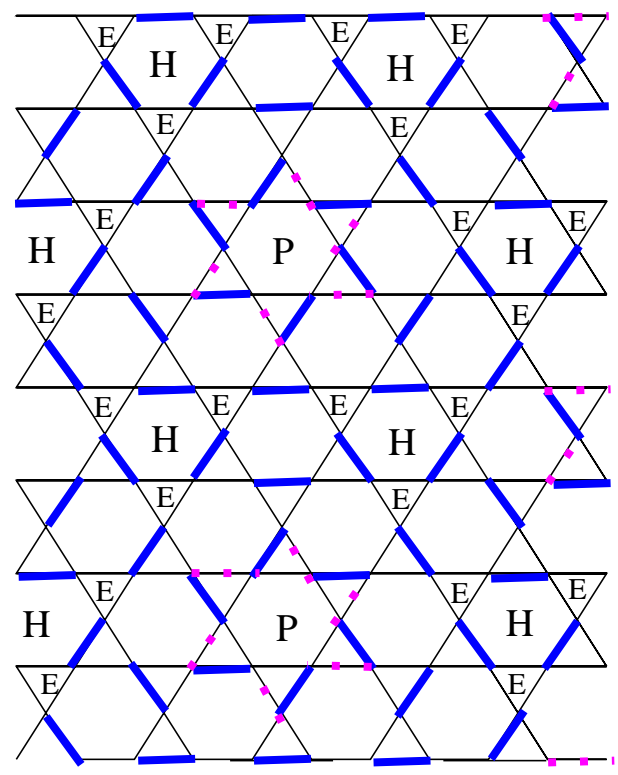

FIG. 1: (Color online) Ground state ordering pattern of low-energy ("strong") bonds (blue) for the Kagome Lattice Heisenberg Model. The perfect hexagons are denoted as H, the empty triangles by $\mathrm{E}$ and the pinwheels as $\mathrm{P}$. The two dimer coverings of the pinwheels that remain degenerate to high orders of perturbation theory are denoted by thick solid (blue) and dotted (magneta) bonds.

linear combinations of VBC ground states [1].

The fact that the 36 -site cluster consists of only a single unit cell of the VBC allows substantial finite-size effects and a strong overlap of the various ground and excited state manifolds. This was evident from our earlier study, where we found that various states of the thermodynamic 
system have energy differences of order $0.001 \mathrm{~J}$ per site, whereas the periodic boundary condition on the 36-site cluster leads to changes in energy of order $0.006 \mathrm{~J}$. This means that there is unlikely to be a definitive signature of the broken symmetry in the spectra of this cluster. It is the purpose of this paper to understand the excitation spectrum of the VBC phase, especially to identify the singlets that fall within the spin gap and to explore their relation to the states obtained in the exact diagonalization study.

The 36-site cluster is large enough that we apply the same linked-cluster methods to develop series expansion for the finite cluster that we used to study the infinite system. Thus both for the infinite system and for the 36site cluster, we have obtained the triplet spectra correct to 7 -th order in the dimer expansion. These series are not long enough to capture the superposition of different ground and excited states in the 36-site cluster, which restore all symmetries of the (undimerized) kagome lattice and lead to states with well defined crystal momenta and point group quantum numbers in the full Brillouin zone. That would require a separate diagonalization study of the model in a restricted basis set [11], which is not part of the calculations presented here. Still, as will see, the series expansion study of triplet and singlet excitations proves quite informative.

The VBC unit cell has 18 dimers. Thus the triplet spectrum is obtained from the eigenvalues of an $18 \times 18$ matrix. For the infinite system, this matrix depends on the momentum in the reduced Brillouin zone of the VBC. Note that all momenta $q$ of the 36 -site cluster map to $q=0$ in the reduced Brillouin zone. The series coefficients are much more convergent for the infinite system than for the finite cluster, consistent with what is expected if the true ground state of the infinite system is a VBC. We estimate the spin gap to be $0.08 \pm 0.02 J$ for the infinite system, with the lowest band of triplets having its minimum at $q=0$. Our series estimates are less accurate for the 36 -site cluster, where we obtain a spin gap $\Delta$ of roughly $0.22 \mathrm{~J}$. The exact diagonalization result for the triplet gap is $0.16419 J[19]$. This difference is within the uncertainties of our calculations of this quantity on the finite cluster.

The two-particle calculations are more difficult, and we have only carried out a general calculation on the 36-site cluster and only to second order; we find as many as 15 different singlet bound states of two triplets. This order is not enough to accurately estimate the absolute energy of these bound states. Just adding up the terms gives a negative energy, in other words, the excitation would fall below the ground state. However, this is also the case for the triplets. For the triplets, where we have longer series, we know that higher-order terms produce positive excitation energies. The expansions for the binding energies appear more reasonable. To second order, the binding energies of the lowest four states, in units of J, are 0.33 , $0.28,0.25$ and 0.25 respectively. These lie between $\Delta$ and $2 \Delta$, implying that these singlet excitations lie within the spin gap. These states together with the ground state can provide up to $5 \times 48=240$ singlet states below the spin gap in the 36-site cluster, roughly consistent with exact diagonalization results.

The plan of this paper is as follows. In section II, we outline the series expansion methods used for the calculations. In section III, we discuss the ground state properties, including the dimerization order parameter. In section IV, we discuss the triplet excitations. In section $\mathrm{V}$, the singlet bound state calculations are discussed. Finally, in section VI, we discuss in more detail the relationship of our work to earlier exact diagonalization studies of the 36-site cluster.

\section{SERIES EXPANSIONS FOR THE VBC PHASE}

We are interested in the properties of the Kagome Lattice Heisenberg Model (KLHM) with Hamiltonian,

$$
H=J \sum_{\langle i, j\rangle} \mathbf{S}_{i} \cdot \mathbf{S}_{j}
$$

where $S_{i}$ are spin-half operators and the sum runs over all nearest-neighbor bonds of the Kagome Lattice. In order to carry out the series expansion study, we write the Hamiltonian as $H_{0}+\lambda H_{1}$, where $H_{0}$ consists of all Heisenberg exchange terms on the strong bonds of the VBC phase and $H_{1}$ consists of all other bonds. Standard Raleigh-Schrodinger perturbation theory in powers of $\lambda$ is used to study the ground state properties of the system [20]. The series are extrapolated to $\lambda=1$ either by summing up terms in the series or by using Pade approximants. To study the triplet excitation spectra, we use the similarity transformation method, first discovered by Gelfand, details for which can be found in the literature [20, 21].

For any finite (or infinite) Lattice, series expansion calculations by the Linked Cluster method require an enumeration of all possible graphs (or clusters) that can be embedded in that lattice up to some size, and a calculation of various recursion relations on those graphs or clusters. We find it convenient to define the graphs by the set of strong bonds (or dimers) that are contained in them, and all weak bonds connecting those strong bonds are included in a single graph. In this sense, our graph enumeration scheme is similar to a low temperature expansion (or strong embedding). The difference between the infinite system and the 36-site system with periodic boundary conditions (PBC) arises from the different graphs that can be embedded in them. In the 36site system there are only 18 dimers, whereas for the infinite lattice their number is infinite, but graphs related by a translation of the unit cell are counted only once. Since the system has a large unit cell, it is important to distinguish between the different inequivalent bonds in the lattice. We have used a simple counting scheme, where all 
connected graphs of the 36-site cluster are treated separately. For the infinite system, all graphs that are distinct under the translation symmetries of the VBC are treated separately. This leads to 19,837 graphs with up to 8 dimers on the 36-site cluster and 67,861 graphs with up to 8 dimers for the infinite Kagome Lattice. While many of these clusters are topologically equivalent, we have not exploited their equivalence. Our calculations are limited by the counting associated with the complex unit cell. These graphs are sufficient for calculating ground state energy correct to 8-th order and other quantities to 7 -th order. Our results for the infinite system and the 36site cluster agree completely with our earlier calculations of the ground state properties to 5 -th order and triplet spectra to second order based on a very small number of specially defined topological graphs, and the longer series continue the trend of surprisingly good convergence for the infinite lattice that we noted earlier [18].

\section{GROUND STATE PROPERTIES: DIMERIZATION ORDER PARAMETERS}

In this section, we discuss the ground state energy and dimerization order parameters for the Kagome Lattice Heisenberg Model. The ground state energy series for the infinite lattice is found to be

$E_{0}^{(\infty)} / J=-\frac{3}{8}-\frac{3}{64} \lambda^{2}-\frac{1}{256} \lambda^{3}-0.00577799 \lambda^{4}-0.000528971 \lambda^{5}-0.000634087 \lambda^{6}+0.0000952526 \lambda^{7}-0.0000470303 \lambda^{8}$.

This leads to an estimate for the ground state energy of $-0.4326 \pm 0.0001 J$. All error bars in this paper are subjective estimates of the uncertainties in series extrapolation to $\lambda=1$. They do not reflect any statistical uncertainties in the calculations. For quantities where we can simply sum up the terms in the series, we estimate their value by their partial sum up to the smallest term in the series (in magnitude) and set the uncertainty to be roughly twice the magnitude of the smallest term. We have checked that the numbers are consistent with Pade approximant estimates.

The dimerization order parameters are defined by the expectation values $\left\langle\mathbf{S}_{i} \cdot \mathbf{S}_{j}\right\rangle$ on the different bonds. Note that the ground state energy implies a mean energy per bond of approximately $-0.216 J$. In the large unit cell, there are many inequivalent bonds. We focus here on the dimerization order parameters inside the "perfect hexagons'. If we consider an isolated hexagon and write our Hamiltonian as $H_{0}+\lambda H_{1}$, with $H_{0}$ containing the exchange on the strong bonds, the symmetry will be restored as $\lambda$ goes to unity and the dimerization order parameter will be zero. In the infinite Kagome Lattice, either the dimerization order parameter inside the 'perfect hexagons' can go to zero, or the coupling with the rest of the lattice (and other hexagons) can generate a selfconsistent dimerization in each hexagon. We find here that the latter case is realized. For the infinite lattice, the series for the expectation value on the strong bond of the 'perfect hexagons' (Bond Type A) is given by

$$
E_{A}=-0.75+0.1875 \lambda^{2}+0.046875 \lambda^{3}+0.0789388 \lambda^{4}+0.00832791 \lambda^{5}+0.00481033 \lambda^{6}-0.0104363 \lambda^{7}-0.00798403 \lambda^{8}
$$

whereas the series for the weak bond in the perfect hexagons (Bond Type B) is

$$
E_{B}=-0.1875 \lambda-0.0703125 \lambda^{2}-0.0683594 \lambda^{3}-0.0106608 \lambda^{4}+0.00889079 \lambda^{5}+0.0172855 \lambda^{6} .
$$

Note that the more difficult off-diagonal expectation values $\left(E_{B}\right)$ are carried out up to only 7-dimer graphs. Summing to the smallest term, the energy of bond type $\mathrm{A}$ is $E_{A}=-0.42 \pm 0.01 J$, and bond type $\mathrm{B}$ is $E_{B}=$ $-0.33 \pm 0.02 J$; the difference is larger if we sum the full series. Note that they are both lower than the mean energy per bond, and that there is a clear alternation inside the perfect hexagons as well, indicating that nonzero VBC order parameters, including dimerization within the perfect hexagons, survive to $\lambda=1$.

It is interesting to compare these results with the Kagome-stripe model, which was studied by DMRG 22]. The latter consists of a single-hexagon wide strip of the Kagome Lattice. That model was found to be spontaneously dimerized in a pattern reminiscent of the "perfect hexagons' in the VBC phase of the KLHM. One important difference is that in the latter model, the threefold rotational symmetry of the Kagome Lattice is absent 
and the dimerization preferably occurs, not surprisingly, along the stripe axis.

We have also extended the ground state energy series for the 36-site cluster. We obtain

$$
E_{0}^{(36)} / J=-\frac{3}{8}-\frac{3}{64} \lambda^{2}-\frac{1}{256} \lambda^{3}-0.00821940 \lambda^{4}-0.00224474 \lambda^{5}-0.000257987 \lambda^{6}+0.00104012 \lambda^{7}+0.00161714 \lambda^{8} .
$$

Summing up to the smallest term leads to the estimate $-0.4365 J$; Pade approximants show large uncertainties due to the strong growth of the last two terms. This should be compared with the exact diagonalization result of $-0.438377 \mathrm{~J}$. It is likely that a large part of the difference is associated with the superposition of different symmetry-broken ground states, an effect which is not captured in our calculations, although it may be the cause of the growth of the last two coefficients.

\section{TRIPLET EXCITATION SPECTRA}

In this section, we study the triplet excitation spectra. As discussed in our earlier paper, an important aspect of the dimer states of the KLHM is the existence of empty triangles, in which there are no strong bonds. All quantum fluctuations originate from the empty triangles. The empty triangles also play a key role in studying the triplet excitations. There are 18 dimers in a unit cell and thus at $\lambda=0$ there are 18 degenerate triplet excitations for every $q$ in the reduced Brillouin zone, all with energy $J$. These triplets can be classified into Light and Heavy particles. Those dimers that border the empty triangles are much more mobile and will be called Light triplets. These are the triplets that make up the lowest energy excitations. There are nine such states per unit cell, residing on the three dimers in each of the two perfect hexagons and on the three dimers that connect two perfect hexagons via empty triangles (the Light Bridging Dimers). A numbering scheme, together with the sign convention for the singlet states on these dimers is shown in Fig. 2. The other nine states are on the 6 pinwheel dimers and on the three dimers parallel to the Light Bridging Dimers (which we call Heavy Bridging Dimers) and lead to heavy or nearly immobile triplets. The latter only indirectly influence the low energy triplets, and that in a very marginal way. Thus, we will focus our discussion here on the 9 Light Dimers. In studying the singlet bound states in the next section, the Heavy Bridging Dimers (see Fig. 2) will also play an important role.

The triplet spectra are studied by the similarity transformation method, which integrates out the multiple triplet states, order by order in perturbation theory, to generate an extended effective Hamiltonian in the subspace of single triplets [20, 21]. Our first task is to analytically study the lifting of the degeneracy in the single triplet subspace. We focus on the lowest lying triplet in
$\Gamma$

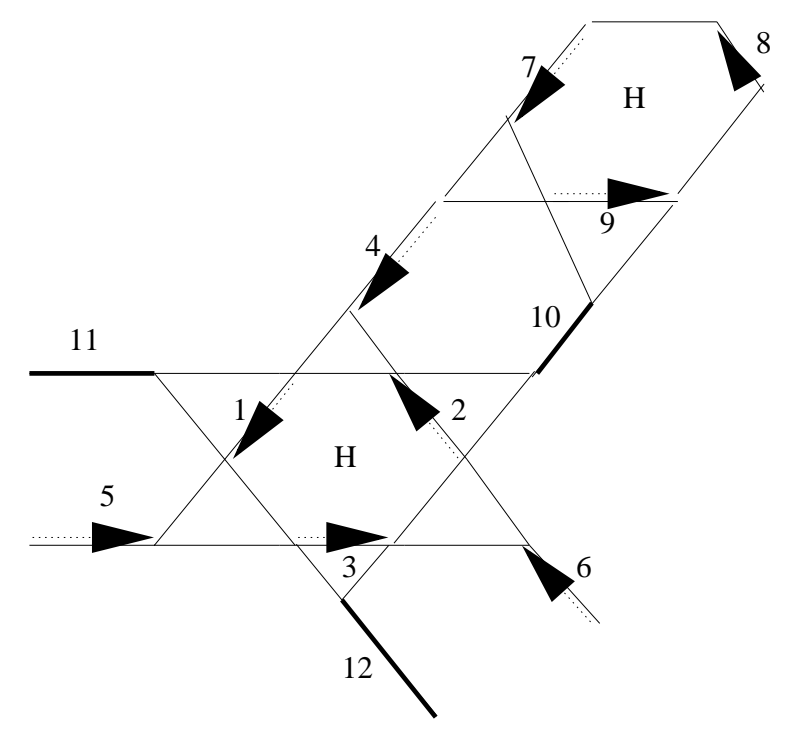

FIG. 2: Labeling of the triplets in a unit cell. The dimers in first perfect hexagon are labeled as 1, 2 and 3 . The Light dimers that bridge the perfect hexagons (Light Bridging Dimers L1, L2 and L3) are labeled as 4, 5 and 6. The dimers in the second perfect hexagon are labeled 7, 8 and 9 . The arrows denote the convention for the singlet states on the bonds. The Heavy dimers between the unit cells (Heavy Bridging Dimers H1, H2, and H3) are shown by thick lines and labeled 10, 11 and 12 .

the reduced Brillouin zone. Once, the degeneracy has been lifted in some order of perturbation theory, we simply need to carry out a non-degenerate perturbation theory for that state. Let $z_{1}=\exp i \vec{k} \cdot \vec{r}_{1}$ with $\vec{r}_{1}=-4 \sqrt{3} \hat{y}$, and $z_{2}=\exp i \vec{k} \cdot \vec{r}_{2}$ with $\vec{r}_{2}=-6 \hat{x}-2 \sqrt{3} \hat{y}$, with the lattice oriented as in Fig. 1, with a nearest-neighbor spacing of unity. In the first order, the effective Hamiltonian is given by $(-\lambda J / 4) M_{1}$, where the matrix $M_{1}$ is shown in Table I, where $z_{3}=z_{1}^{*} z_{2}$. Note that the matrix depends on conventions of the unit cell and of the choice of arrows, which define the singlet states on the bonds. Note also that we have changed notation from our previous paper [18], to make it more symmetric.

For $q=0$ the matrix $M_{1}$ leads to 4 degenerate states. Two of these are triplets localized within a 'perfect hexagon'. These are invariant under the $2 \pi / 3$ rotational symmetry of the lattice, the only symmetry that remains unbroken in the VBC. The other two states form a dou- 
TABLE I: Matrix $M_{1}$

\begin{tabular}{rrrrrrrrr}
\hline \hline 0 & -1 & -1 & -1 & 1 & 0 & 0 & 0 & 0 \\
-1 & 0 & -1 & 1 & 0 & $-z_{1}$ & 0 & 0 & 0 \\
-1 & -1 & 0 & 0 & -1 & $z_{1}$ & 0 & 0 & 0 \\
-1 & 1 & 0 & 0 & 0 & 0 & -1 & 0 & 1 \\
1 & 0 & -1 & 0 & 0 & 0 & 0 & $z_{3}$ & $-z_{3}$ \\
0 & $-z_{1}^{*}$ & $z_{1}^{*}$ & 0 & 0 & 0 & 1 & -1 & 0 \\
0 & 0 & 0 & -1 & 0 & 1 & 0 & -1 & -1 \\
0 & 0 & 0 & 0 & $z_{3}^{*}$ & -1 & -1 & 0 & -1 \\
0 & 0 & 0 & 1 & $-z_{3}^{*}$ & 0 & -1 & -1 & 0 \\
\hline \hline
\end{tabular}

blet that have a nonzero 'angular momentum' under this rotation. By symmetry, the former pair of two states can not mix with the latter. Higher order calculations show that the nonzero angular momentum states have lower energy starting in 3rd order. This is also what happens on the 36-site cluster, which only has $q=0$ in the reduced Brillouin zone. The series for the lowest triplet (the spin gap) on the infinite-lattice is

$$
\Delta_{\infty} / J=1-0.5 \lambda-0.875 \lambda^{2}+0.440625 \lambda^{3}+0.074479167 \lambda^{4}-0.04346788 \lambda^{5}-0.02336301 \lambda^{6}-0.1487368 \lambda^{7}
$$

For the 36-site cluster the series for the lowest triplet is

$$
\Delta_{36} / J=1-0.5 \lambda-0.875 \lambda^{2}+0.440625 \lambda^{3}+0.4864583 \lambda^{4}-0.1698405 \lambda^{5}-0.4289909 \lambda^{6}+0.393891 \lambda^{7}
$$

Note that the difference first appears in 4 th order, just as for the ground state, as the wrapping around of the $\mathrm{PBC}$ begins to influence the state. Clearly, the series for the infinite lattice is better behaved, although the last term suggests that one does not have absolute convergence. We can speculate that the lack of convergence might arise from the process of lifting of the degeneracy in low orders and this can have a 'ringing' effect at higher orders. Using Pade approximants, we estimate the spin gap for the infinite system to be $\Delta_{\infty}=0.08 \pm 0.02 \mathrm{~J}$.

For the 36-site cluster, Pade approximants lead to an estimate of the gap of approximately $0.22 \mathrm{~J}$. This should be compared with the exact-diagonalization result of $0.16419 \mathrm{~J}$. Given the irregularity in this series, this is within the uncertainties of the calculation. It is also likely that the superposition of different symmetrybroken states that must appear in the finite-size cluster at $\lambda=1$ may lead to a further lowering of the spin gap. For the ground state this extra energy is of order $0.002 \mathrm{~J}$ per lattice site, so the total energy difference for the 36site cluster is of order $0.07 \mathrm{~J}$. One might expect energy corrections of this order for the excitations as well.

For $q \neq 0$, there are only 3 states that are degenerate at order $\lambda$. Two of them are localized in the "perfect hexagons'. The third is given by the vector in Table II, and is delocalized. Once again, in third-order perturbation theory, the delocalized state ends up with the lower energy. On the infinite lattice, the difference between the lowest energy excitation at any $q$ and that at $q=0$ remains very small in all orders of the calculation that we have studied so far. We find a very small band width of order $0.01 \mathrm{~J}$, with the band minimum remaining at $q=0$.

We note that in addition to the lowest-lying triplet band studied here, there is a second low-energy band,
TABLE II: Nontrivial eigenvector of matrix $M_{1}$

$$
\begin{array}{r}
\hline \hline z_{1}+z_{1} z_{3}-2 z_{3} \\
z_{1} z_{3}+z_{3}-2 z_{1} \\
z_{3}+z_{1}-2 z_{1} z_{3} \\
3\left(z_{1}-z_{3}\right) \\
3\left(z_{3}-z_{1} z_{3}\right) \\
3\left(z_{3}-1\right) \\
z_{1}+1-2 z_{3} \\
z_{3}+z_{1}-2 \\
1+z_{3}-2 z_{1} \\
\hline \hline
\end{array}
$$

which is more dispersive and is degenerate with the lowest band at $q=0$. Then there are at least two more low-lying triplet bands consisting of triplets which are nearly localized inside a perfect hexagon and weakly hop from one perfect hexagon to next. In contrast to the lowest triplet, the series expansions for these latter states are poorly convergent for the infinite lattice, perhaps reflecting the fact that before we reach $\lambda=1$ they become unstable and can decay into the lower triplet and even lower-lying singlets.

\section{SINGLET EXCITATIONS}

In this section, we discuss the singlet bound states of two triplets. We focus only on the 36 -site cluster, which means that the two triplets are restricted to a single unit cell with periodic boundary conditions. The bound states can be divided into two types: (1) Light-Heavy bound states and (2) Light-Light bound states. 


\section{A. Light-Heavy Bound states}

The light-heavy bound states arise from the fact that when the heavy triplet lies on one of the Heavy Bridging Dimers (10, 11 or 12 in Fig. 2) and the light triplet lies on one of its neighboring dimers in one of the 'perfect hexagons' they have an attraction of magnitude $-\lambda J / 2$. However, in order $\lambda$ the light triplet can also move around. Hence, to calculate the singlet bound state and binding energy correct to order $\lambda$ we need to once again consider a 9-dimensional subspace, where the heavy triplet is held fixed but the light triplet is allowed to hop between the nine light dimers. This is very much like the matrix $M_{1}$. The only difference is that when the light and heavy particles share a pair of common weak bonds there is a diagonal binding energy of $-\lambda J / 2$. Diagonalizing the $9 \times 9$ matrix, we find two bound states. In the more tightly bound state the wavefunction is symmetric under the reflection that exchanges the two ends of the occupied heavy dimer, whereas it is antisymmetric for the other bound state. Since the heavy triplet can be in 3 equivalent positions, this implies a total of six light-heavy bound states on the 36-site cluster.

To get the binding energies of the states to second order, we have calculated the $9 \times 9$ effective Hamiltonian to second order given by

$$
\mathcal{H}_{e f f}=P H_{1}(1-P) \frac{1}{E_{2}-H_{0}}(1-P) H_{1} P,
$$

where $P$ is the projection operator on the two-particle subspace and $E_{2}=2 J$ is the bare energy of the twoparticle states. Calculating the expectation value of the effective Hamiltonian in the bound states obtained in first order, leads to energies to second order for the bound states, relative to the ground state, of

$$
E_{l h 1}=2-1.22545 \lambda-0.88485422 \lambda^{2},
$$

and

$$
E_{l h 2}=2-1.171040 \lambda-0.83436254 \lambda^{2} .
$$

Note that if we set $\lambda$ to unity, this would mean a negative energy for these states, i.e., an energy even below the ground state. However, the second order calculation of the minimum triplet energy is $1-0.5 \lambda-0.875 \lambda^{2}$. Thus, if we simply add up the terms at $\lambda=1$, these states do not even remain bound. Clearly a higher order calculation is needed to get an estimate of the energy of these lightheavy states. That is beyond the scope of the current work.

For the infinite system, the Light-Heavy bound state calculation at order $\lambda$ is a tight-binding model for the light triplet with a short-range impurity potential near the site of the heavy triplet. The light triplet can go arbitrarily far away from the heavy triplet, thus the calculation requires diagonalizing an infinite-dimensional matrix already at order $\lambda$. It seems clear that the light-heavy bound state is nearly dispersionless. For the state to develop any $q$-dependence the heavy triplet would have to move from one unit cell to another and that is a very high order process.

\section{B. Light-Light Bound states}

When two light triplets share a weak bond they also feel an attraction in the singlet sector in order $\lambda$ of magnitude $-\lambda J / 4$. To study the light-light bound state fully in order $\lambda$, we need to consider the $C_{2}^{9}=36$-dimensional Hilbert space in which the two light triplets occupy any two of the 9 light dimers. In this case, the diagonalization of the 36-dimensional matrix leads to 8 different bound states.

We have also calculated the energy of these bound states to order $\lambda^{2}$. The energies of various bound states to second order are given in Table III. Note that at order $\lambda^{2}$ the light-light bound states have significantly lower energies than the light-heavy bound states. If we simply add up the terms in the series we will get strongly negative results, which are unphysical. However, it is quite plausible that these large negative energies simply follow from the large negative energies for individual triplets at this order, which we now know are corrected at higher orders. Thus we look at the series for the binding energy by subtracting at each order the energy of a bound state from the lowest energy of two 'free' triplets.

TABLE III: Series Coefficients for the energies of the LightLight Bound states to order $\lambda^{2}$

\begin{tabular}{rrrr}
\hline \hline state & $\lambda^{0}$ & $\lambda^{1}$ & $\lambda^{2}$ \\
1 & 2 & -1 & -2.08333 \\
2 & 2 & -1.22554 & -1.81451 \\
3 & 2 & -1.08013 & -1.92010 \\
4 & 2 & -1.08013 & -1.92010 \\
5 & 2 & -1.15062 & -1.71371 \\
6 & 2 & -1.15062 & -1.71371 \\
7 & 2 & -1.14039 & -1.73862 \\
8 & 2 & -1.14039 & -1.73862 \\
9 & 2 & -1.14039 & -1.68347 \\
\hline \hline
\end{tabular}

Simply evaluating the series at $\lambda=1$, we obtain binding energies for these states of $0.33,0.29,0.25,0.25,0.13$, $0.13,0.11,0.11$ and -0.03 times $J$, respectively. So 8 of the 9 states are estimated to remain bound. Furthermore, the binding energy of the lowest four states exceeds the spin gap $\Delta_{36}$ and this suggests that these singlet states will fall below the lowest triplet. While these secondorder calculations cannot be taken as quantitatively accurate estimates for the energies of these singlet bound states, our calculations strongly suggest that there are lots of singlet states which can fall within the spin gap. Since each state corresponds to 48 distinct states when the symmetry-related ground states are counted on the 
36-site cluster, these 4 singlet excitations together with the ground state can lead to $48 \times 5=240$ singlet states within the spin gap. This is clearly a correct order of magnitude when compared to the exact diagonalization. The nature of the singlet bound states is discussed in the next section.

For the infinite system, the light-light bound states can be dispersive, as each of the triplets is dispersive. However, given that the light triplets have a very weak dispersion, the dispersion of the bound state is likely to also be weak. Our calculations, thus, suggest that there are a few low-energy bands of one or more weakly dispersive singlet excitations. In the future, we hope to develop systematic methods to study these singlet excitations and their dispersion to higher order.

\section{DISCUSSIONS: NATURE OF SINGLETS AND RELATION TO EXACT DIAGONALIZATION STUDIES}

In this section, we first discuss qualitatively the wavefunctions of the singlet bound states in the order they are listed in Table III:

\section{A. Wavefunctions and symmetries of bound states}

1. The state with largest binding energy when truncated at order $\lambda^{2}$ has a very simple wavefunction. One triplet is in one perfect hexagon and the other triplet is in the other perfect hexagon. The two triplets attract each other by a very unusual interference mechanism. Since they are never on neighboring sites they have no direct contact attraction and thus no binding at order $\lambda$. The attraction arises from the fact that when the two triplets are on dimers separated by a light bridging dimer that shares a weak bond with both, then there is constructive interference to go to a three-triplet state and back, and this process significantly lowers the energy of this bound state in order $\lambda^{2}$.

2. The second largest binding energy is for a state where both triplets are mostly in the same perfect hexagon, and the state is symmetric under the exchange of perfect hexagons.

3-6. A set of two separate doubly degenerate states (3,4 and 5,6 in Table III) are the ones with nonzero 'angular momentum'. In these, there is significant probability of finding one or both triplets to be outside the perfect hexagons (on the light bridging dimers). The probability of finding both triplets in same perfect hexagon is very small. Since these states are bound states of what in third order become the lowest-energy triplets, and as they will not have any level repulsion from the ground state due to having different symmetry, it seems quite possible that finally the lower of these bound states end up with the lowest energy overall.
7-9. Finally there are a set of 3 states which are degenerate in order $\lambda$ but split into a doublet and a singlet in order $\lambda^{2}$. Their wavefunction has significant probability of finding the two triplets in the same perfect hexagon, but the wavefunction is antisymmetric under the exchange of the two hexagons.

An important question is precisely how are the singlet bound states studied here related to the low-lying singlet states found in the exact diagonalization studies. This issue cannot be quantitatively addressed based on series expansion alone. What we cannot address here is what happens when the 48 different ground states corresponding to the symmetry-related dimer patterns are fully admixed at $\lambda=1$. That is what restores the full symmetry of the underlying kagome lattice, and leads to states which have well-defined quantum numbers under all the symmetries of the lattice. To explore this issue further, it may be useful to study the KLHM in a reduced basis set consisting of the various low-lying states that we have found, and their symmetry partners.

We also note that low-lying singlets found in our study include some with nonzero 'angular momentum' and thus lower symmetry than the honeycomb VBC, consistent with what is found in the exact diagonalization studies. It is also important to note that the two-particle bound states generally lie outside the nearest-neighbor singlet dimer subspace of the Heisenberg model.

\section{B. Relevance to Experiments}

Recently, there has been much interest in the material $\mathrm{ZnCu}_{3}(\mathrm{OH})_{6} \mathrm{Cl}_{2}$, as it represents a first example of structurally perfect spin-half Kagome Lattice [23, 24, 25, 26]. Unfortunately, this material may have significant antisite disorder leading to magnetic impurities and possibly also has significant Dzyloshinski-Moria (DM) interactions [27, 28]. Since it has a spin-gap in its spectrum, the VBC state might survive a sufficiently small DM interaction. Thus it is important to obtain samples with reduced impurities and ascertain the strength of DM interactions.

If the $\mathrm{VBC}$ phase is relevant to a material, Raman scattering can be used to study the singlet states just as Neutron scattering is used to study the triplet spectra. Even if there is short-range VBC order, low lying singlet excitations may be sharply defined. Raman scattering on $\mathrm{ZnCu}_{3}(\mathrm{OH})_{6} \mathrm{Cl}_{2}$ should be used to look for low-lying singlet states.

In the future, it would be useful to develop a more detailed phenomenological picture of the experimental properties in the VBC phase. That is distinct from the goals of the present work. 


\section{Acknowledgments}

We would like to thank Philippe Sindzingre for several useful communications. This work was supported by the US National Science Foundation, Grants No. DMR0240918 (R. S.) and DMR-0213706 (D. H.) and PHY0551164.
[1] For a recent review see C. Lhuillier, arXiv:cond-mat/0502464.

[2] C. Zeng and V. Elser, Phys. Rev. B 42, 8436 (1990).

[3] V. Elser, Phys. Rev. Lett. 62, 2405 (1989).

[4] R. R. P. Singh and D. A. Huse, Phys. Rev. Lett. 68, 1766 (1992).

[5] P. W. Leung and V. Elser, Phys. Rev. B 47, 5459 (1993).

[6] N. Elstner and A. P. Young Phys. Rev. B 50, 6871 (1994).

[7] P. Lecheminant, B. Bernu, C. Lhuillier, L. Pierre and P. Sindzingre, Phys. Rev. B 56, 2521 (1997).

[8] F. Mila, Phys. Rev. Lett. 81, 2356-2359 (1998).

[9] C. Waldtmann, H. U. Everts, B. Bernu, C. Lhuillier, P. Sindzingre, P. Lecheminant and L. Pierre, Eur. Phys. J. B 2, 501 (1998).

[10] G. Misguich and B. Bernu, Phys. Rev. B 71, 014417 (2005).

[11] G. Misguich and P. Sindzingre, J. Phys.: Condens. Matter 19, 145202 (2007).

[12] J. B. Marston and C. Zeng, J. Appl. Phys. 69, 5962 (1991).

[13] A. V. Syromyatnikov and S. V. Maleyev, Phys. Rev. B66, 132408 (2002).

[14] P. Nikolic and T. Senthil, Phys. Rev. B 68, 214415 (2003).

[15] R. Budnik and A. Auerbach, Phys. Rev. Lett. 93, 187205 (2004).

[16] M. Hermele, T. Senthil, and M. P. A. Fisher, Phys. Rev. B 72, 104404 (2005).

[17] Y. Ran, M. Hermele, P. A. Lee, and X. -G. Wen, Phys. Rev. Lett. 98, 117205 (2007).

[18] R. R. P. Singh and D. A. Huse, Phys. Rev. B 76, 180407
(2007).

[19] P. Sindzingre, G. Misguich, C. Lhuillier, B. Bernu, L. Pierre, Ch. Waldtman, H-U Everts, Physical Review Letters, 84, 2953 (2000); P. Sindzingre and C. Lhuillier, to be published.

[20] M. P. Gelfand and R. R. P. Singh, Adv. Phys. 49, 93 (2000); M. P. Gelfand, R. R. P. Singh and D. A. Huse, J. Stat. Phys. 59, 1093 (1990).

[21] J. Oitmaa, C. Hamer and W-H. Zheng, Series Expansion Methods for strongly interacting lattice models, (Cambridge University Press 2006).

[22] S. R. White and R. R. P. Singh Phys. Rev. Lett. 85, 3330 (2000).

[23] J. S. Helton, K. Matan, M. P. Shores, E. A. Nytko, B. M. Bartlett, Y. Yoshida, Y. Takano, A. Suslov, Y. Qiu, J.-H. Chung, D. G. Nocera, and Y. S. Lee, Phys. Rev. Lett. 98, 107204 (2007).

[24] O. Ofer, A. Keren, E. A. Nytko, M. P. Shores, B. M. Bartlett, D. G. Nocera, C. Baines, and A. Amato, cond-mat/0610540

[25] P. Mendels, F. Bert, M. A. de Vries, A. Olariu, A. Harrison, F. Duc, J. C. Trombe, J. Lord, A. Amato, and C. Baines, Phys. Rev. Lett. 98, 077204 (2007).

[26] T. Imai, E. A. Nytko, B. M. Bartlett, M. P. Shores, D. G. Nocera, cond-mat/0703141

[27] M. Rigol and R. R. P. Singh, Phys. Rev. Lett. 98, 207204 (2007).

[28] G. Misguich and P. Sindzingre, Eur. Phys. J. B 59, 305 (2007). 